\title{
Revision rhinoplasty for contracted nose in Asia
}

\author{
Huy Tien Vo, MD \\ Kangnam Aesthetic Hospital, Hanoi, Vietnam
}

Contracted nose is a deformity, occurring both internally and externally, of the nose due to scar tissue contraction or capsular formation after repeated rhinoplasties using silicone implants. To comprehensively address the issue, the following surgical techniques were chosen: releasing capsular contracture by dual plane dissection when undermining the skin, and elongating the framework by septal extension graft or autogenous rib cartilage graft. From 2013 to 2017, 253 patients with contracted nose underwent combined procedures: dual plane dissection, extended spreader graft \& caudal septal extension graft, total septal reconstruction by autogenous rib cartilage, tip repositioning and tip grafts, and dorsal augmentation. Satisfactory results were obtained in 240 (95\%) patients. The results included extended skin and lengthened alar cartilage, elongated framework, and lengthened and projected nasal tip. Thirteen patients $(5 \%)$ who underwent short-nose correction required revision surgeries because of overcorrection or undercorrection of nasal length, poor tip projection, or inadequate correction of columellar retraction. The length of the nose could be extended by dual plane dissection to release adhesion of skin and scar tissue, extended spreader grafting, dorsal augmentation, and tip and alar grafting. The goal of surgery is to use to use a combination of techniques to achieve the aesthetic goals for both patients and surgeons.

Keywords: combined procedures; contracted nose; revision rhinoplasty; rhinoplasty

\section{Introduction}

Contracted nose is a deformity from both the inside and outside the nose caused by the contraction of scar tissues or the formation of capsules after a patient has undergone repeated rhinoplasties or surgeries with silicone implants [1]. The deformities include: having a decreased nasal length with an abnormally increased nostril show, or a cephalically rotated nasal tip with a more obtuse nasolabial angle, etc. [2].

There are many causes of nasal contracture. In Asia, many patients have undergone multiple rhinoplasty operations, especially with the use of silicone implants [3]. The excessive scar tissues or capsular contracture around the implant can develop into contracture of the nose. Infection is another cause of nasal contracture, and it could lead to an even more serious contrac- ture if the patient has undergone multiple previous rhinoplasties (Fig. 1, 2).

A shortened nose could also be caused by the contracture of alar cartilage (Fig. 3). The alar is pulled up cephalically, and the nasal tip is lifted in the forehead direction resulting in the contracture and deformity of the nostrils. Moreover, depression of the nose is caused by collapsed alar cartilages, pinched nasal tip, collapsed internal and external valves, or nasal obstruction.

Nasal deformity can also occur due to contracture of the soft tissue (Fig. 4). It can be the result of damaged nasal structure due to previous surgeries or infection, excessive scar tissues inside the nasal skin, or limited type of extractable graft cartilage being used up from previous operations. There are many challenges associated with the correction of contracted nose, for example: tight skin due to scar-capsule adhesion, scar tissues around the

Received March 4, 2019; Revised June 20, 2019; Accepted June 20, 2019

Corresponding author: Huy Tien Vo

E-mail: bsvohuy@gmail.com, ORCID: https://orcid.org/0000-0002-7821-4135

This is an Open Access article distributed under the terms of the Creative Commons Attribution Non-Commercial License (http://creativecommons.org/licenses/by-nc/4.0), which permits unrestricted non-commercial use, distribution, and reproduction in any medium, provided the original work is properly cited.

Copyright (c) 2019 Korean Society of Korean Cosmetic Surgery and medicine (KSKCS \& KCCS). 


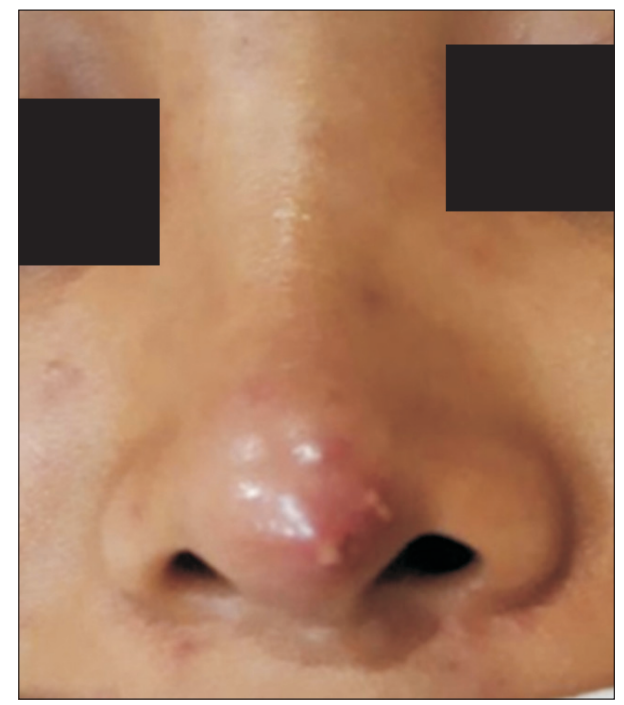

Fig. 1. Infection leads to nasal contracture.

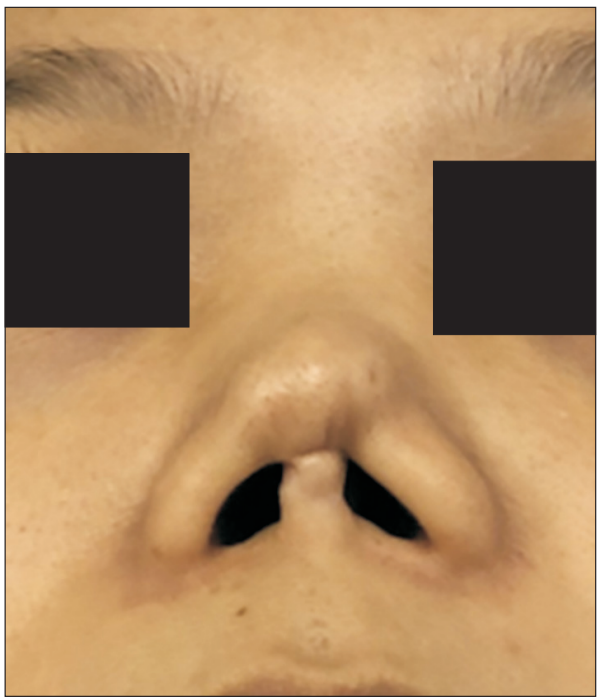

Fig. 2. Contracture caused by previous silicone implant.

cartilage framework, techniques to lengthen the nasal tip (most post-surgical contracted nose has both structural problems such as weak upper and lower lateral cartilage, septum cartilage, leading to the difficulty in adding septal extension graft to caudal septum). With all the reasons combined with associated difficulties mentioned above, contracted nose correction becomes one of the most difficult procedures in Asian rhinoplasty.

\section{Case report}

\section{Strategy for correction of contracted nose}

The following criteria were evaluated to determine the degree

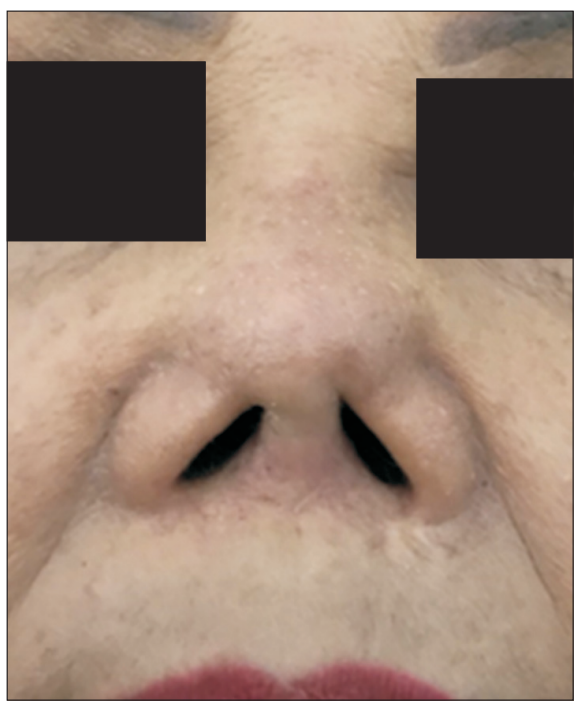

Fig. 3. Deformity due to the contracture of alar cartilage.

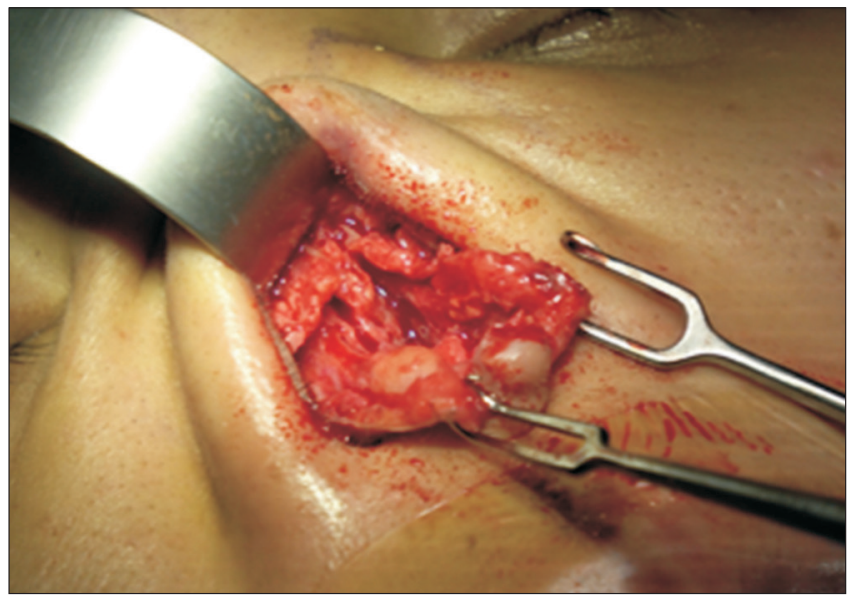

Fig. 4. Deformity due to the contracture of the soft tissue.

of nasal contracture: prior operative history, skin mobility, capsule contracture, framework, nasal septum, degree of cephalic rotation of the nasal tip, alar \& columella positions, and the amount of available cartilage graft used. For framework elongation, the proper surgical techniques included uni- or bi-lateral extended spreader graft and caudal septal extension graft, and total septal reconstruction. Additionally, to release the contracted tissues and raise the nasion superiorly, the skin and tipdefining point has to be lengthened. A firm structural support was required to reinforce and lengthen all three legs of the "tripod" of the nasal tip. The correct material for revision rhinoplasty was also carefully considered to avoid complications. The chosen materials included: nasal septal cartilage, ear cartilage, autogenous rib cartilage, Gore-tex (PTEE), or other alloplastic 
implants.

In short, the surgical techniques for contracted nose correction operation include:

- Incision: open rhinoplasty incision, or on previous scar in the columella

- Skin undermining: dual plane dissection

- Elongating the framework: harvest of septal cartilage, septal extension graft, or use of autogenous rib cartilage

- Tip repositioning and tip grafts

- Dorsal augmentation

- Alar contour graft or lateral crural graft

- Composite graft and skin closure

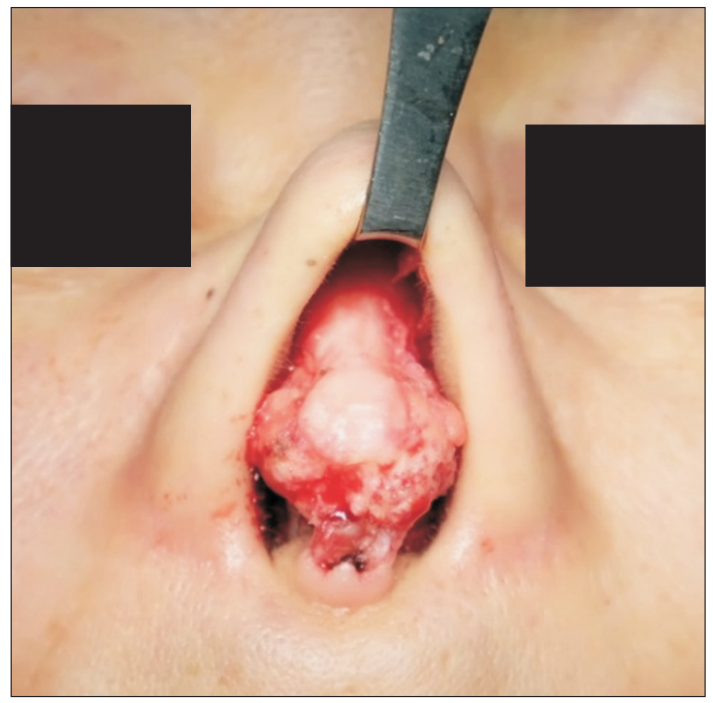

Fig. 5. Superficial plane dissection.

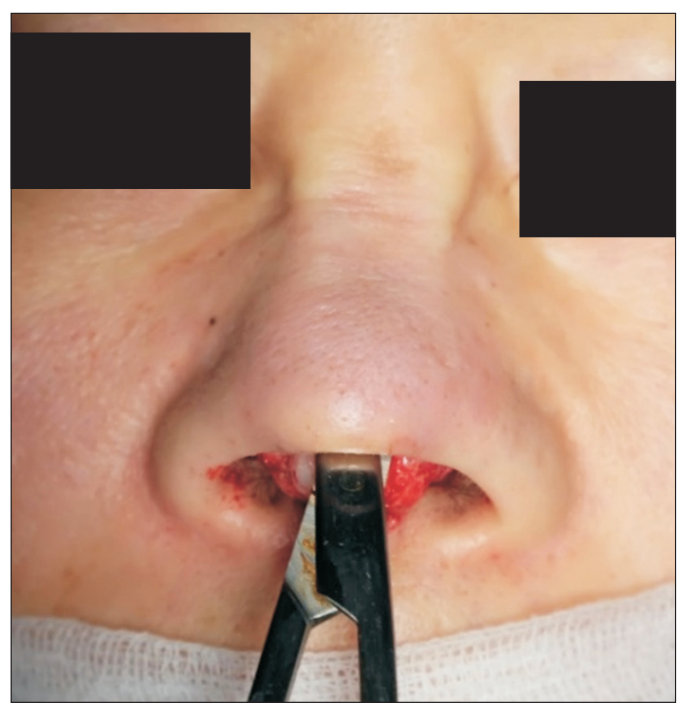

Fig. 6. Superficial plane dissection.

\section{Surgical techniques}

From 2013 to 2017, 253 patients with contracted nose underwent combined procedures: dual plane dissection, extended spreader graft \& caudal septal extension graft, total septal reconstruction by autogenous rib cartilage, tip repositioning and tip grafts, and dorsal augmentation.

\section{Dual plane dissection technique}

The dual plane dissection technique is an important process in contracted nose correction because the scar tissue makes the skin lengthening procedure difficult. In short nose correction operations, both the skin and alar cartilage need to be extended,

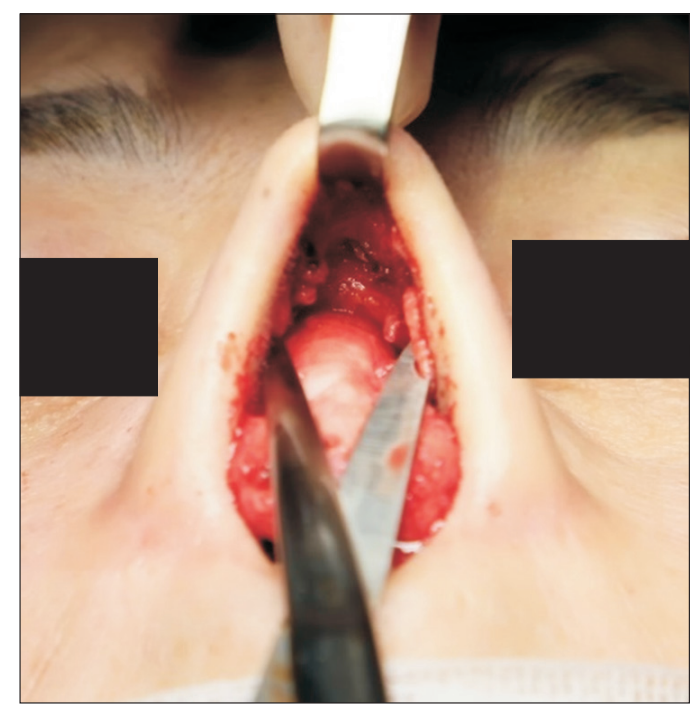

Fig. 7. Superficial plane dissection.

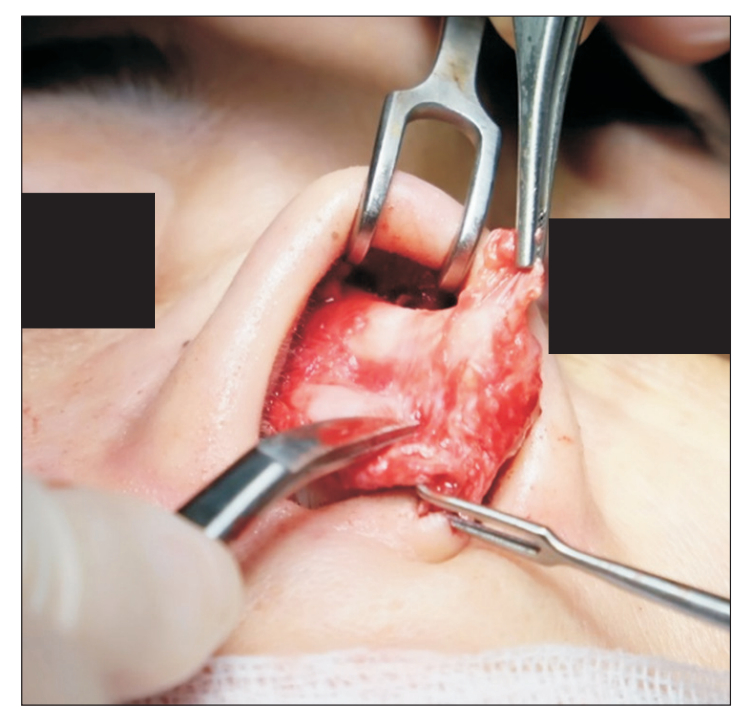

Fig. 8. Deep plane dissection. 
in addition to the septum cartilage graft. Dissection must fully release the adherence between skin-scar / capsule flap-deep planes (periosteum).

\section{Superficial plane dissection}

Superficial skin dissection starts between the skin, scar tissue, and contracted capsule (Fig. 5, 6). The dissection should be made as wide as possible depending on the mobility of the skin (Fig. 7). It must also not inure the integrity of the skin and irregularity of the plane (not too much thin skin).

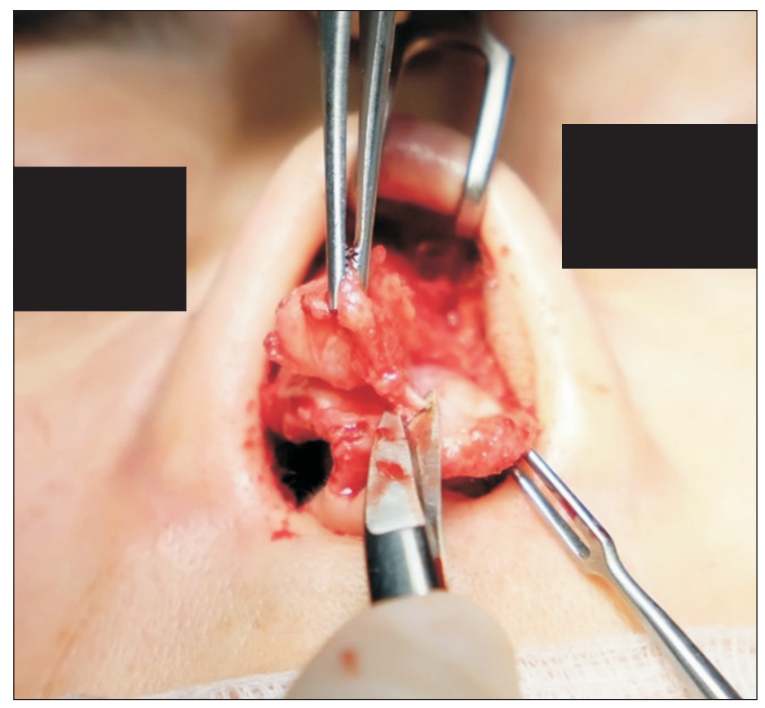

Fig. 9. Deep plane dissection.

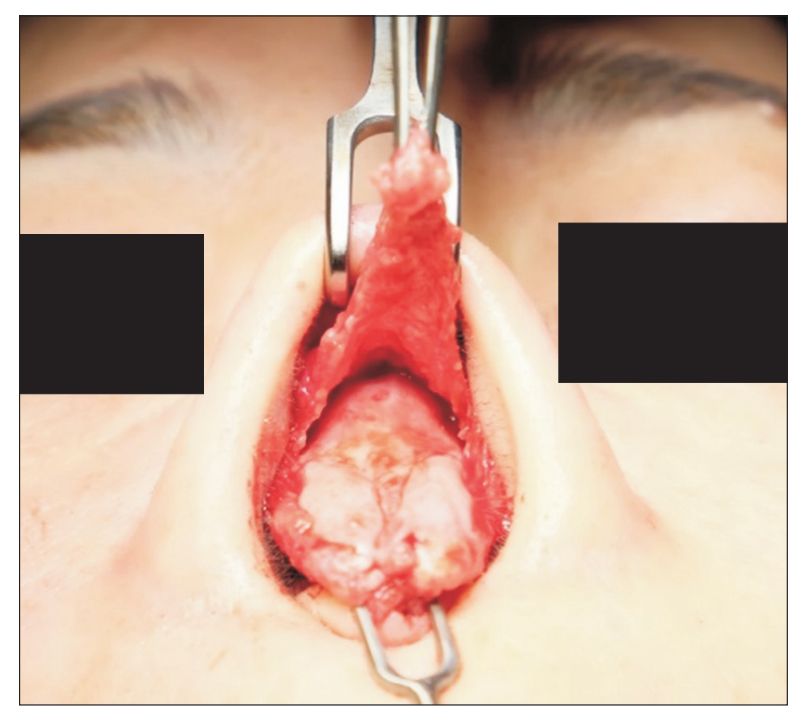

Fig. 10. Deep plane dissection.

\section{Deep plane dissection}

The dissection starts from the incision line of the columella toward the medial crus, to the lateral crus and dorsum cartilage, and then the radix. The dissection needs to separate scar/capsule tissues (Fig. 8-10).

\section{Releasing lower lateral cartilages}

The purpose of releasing the lower lateral cartilages is to fully release the fibrous connective tissues between the lower lateral cartilages, upper lateral cartilages, and suspensory ligament. The scar tissue that limits the movement of alar cartilage in contracted nose, even in membranous septum, must be released. Disconnecting the accessory ligament after dissecting around

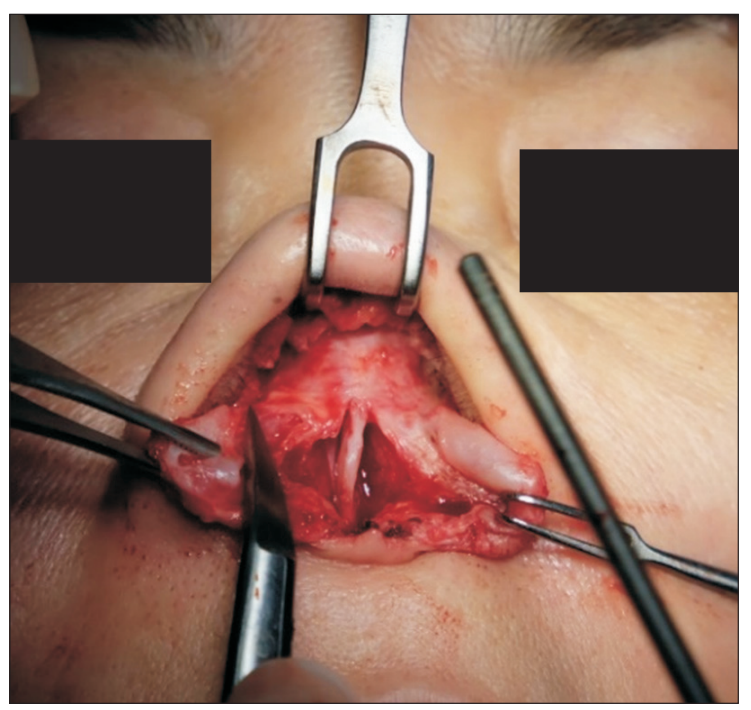

Fig. 11. Lower lateral cartilage release.

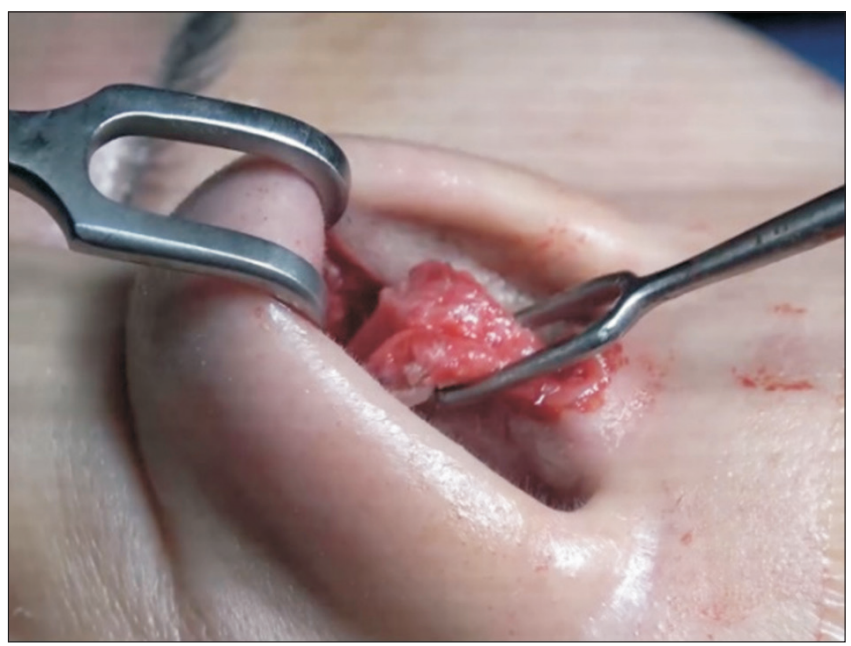

Fig. 12. Lower lateral cartilage release. 


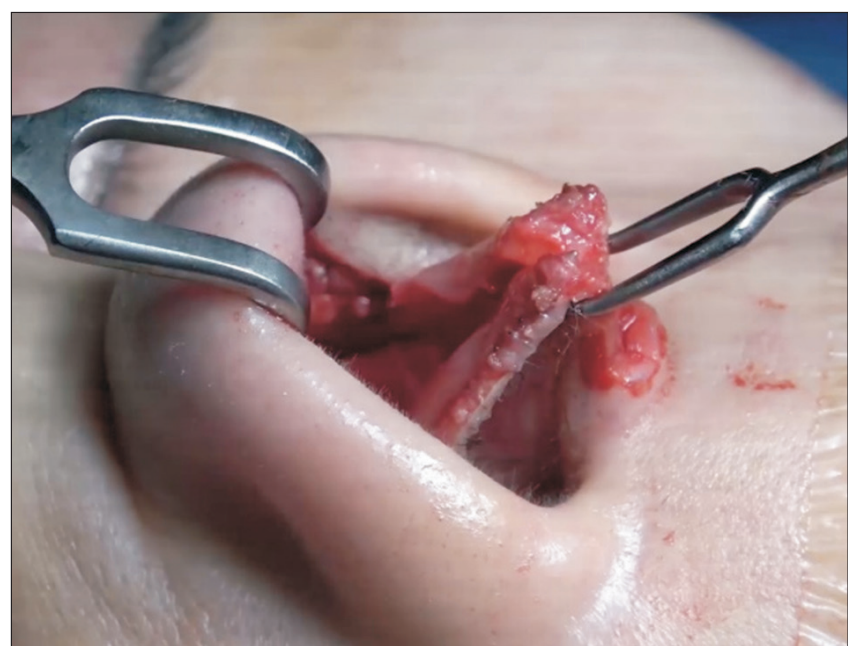

Fig. 13. Lower lateral cartilage release.

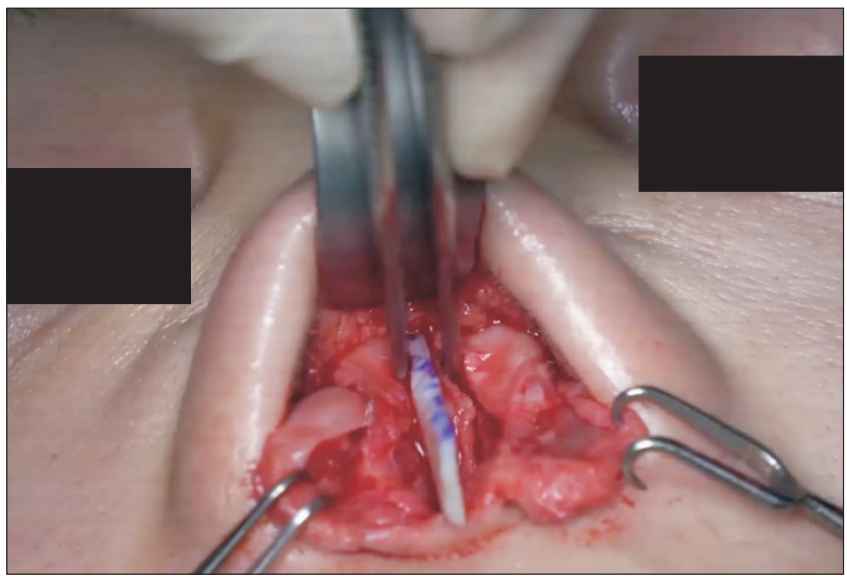

Fig. 14. Elongating the framework.

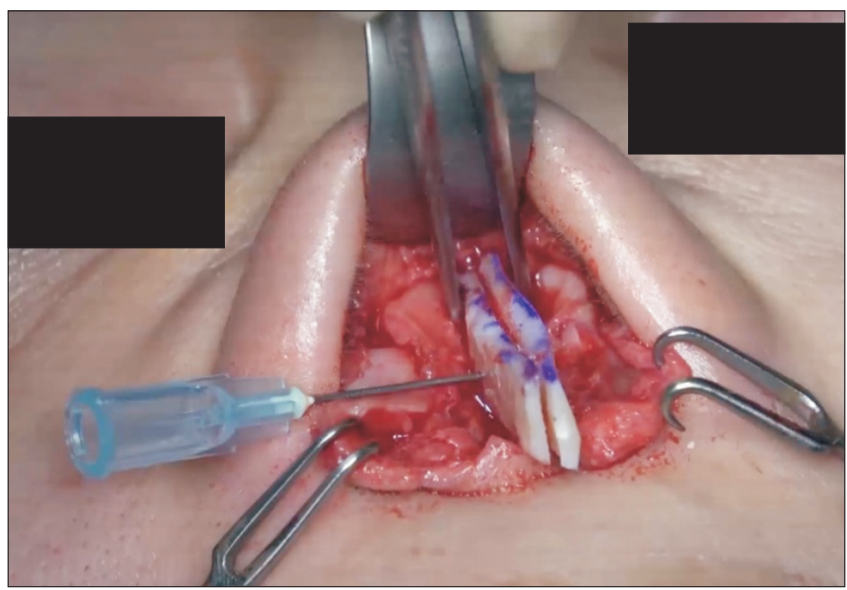

Fig. 15. Elongating the framework. the hinge complex helps improve the mobility of the alar cartilage (Fig. 11-13).

\section{Elongating the framework}

Spreader grafts are added on each side, extending beyond the anterocaudal septum proportional to the required elongation. Then, they are fixed to the septum using 3 mattress sutures of 5-0 PDS.

A columella strut is placed in position that has a width equal to the required elongation of the nose plus the width of the medial crura (Fig. 14-16). Finally, the medial crura are sutured to the newly placed strut in at least 2 places (Fig. 17).

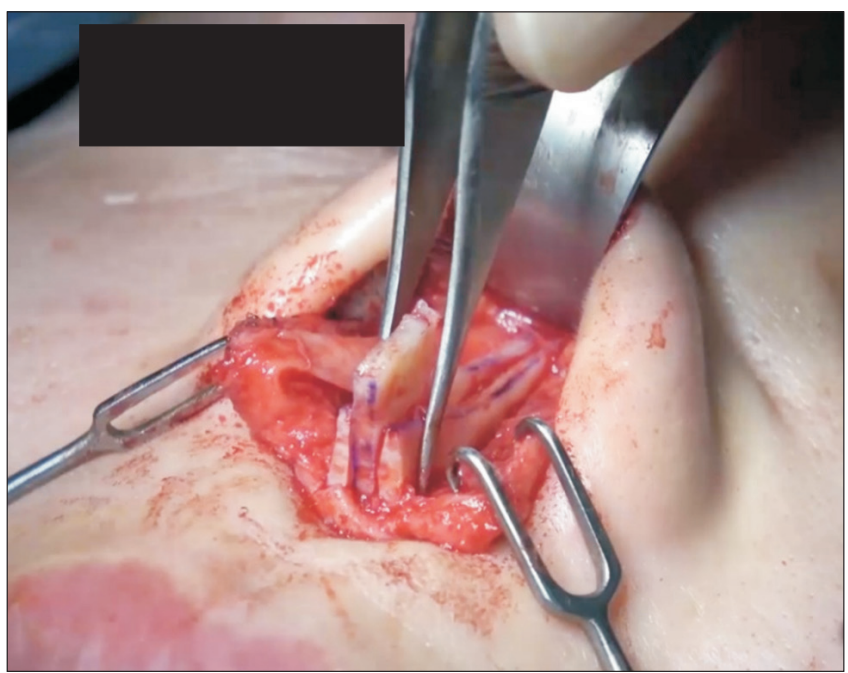

Fig. 16. Elongating the framework.

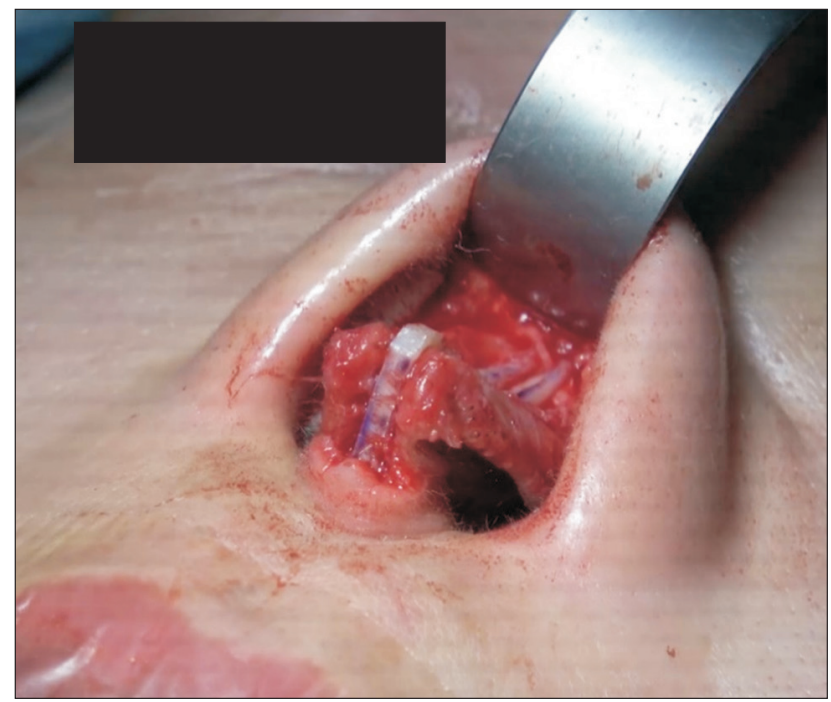

Fig. 17. Elongating the framework. 


\section{Autogenous rib cartilage}

There is the presence of septal perforation if the patient has had harvest of the septal cartilage in the past, previous submucosal resection or septoplasty, or fractured nasal septum. The rib cartilages are usually harvested at the 6th and 7th, or 8th rib cartilage. They should be harvested as much as possible, especially if they are used for dorsal onlay graft. Perichondrium also needs to be harvested enough to cover the dorsum and nasal tip.

\section{$L$-shape reconstruction}

Lengthening of the nose with an L-shaped reconstruction is based on dorsal onlay graft with a columellar strut and fixation

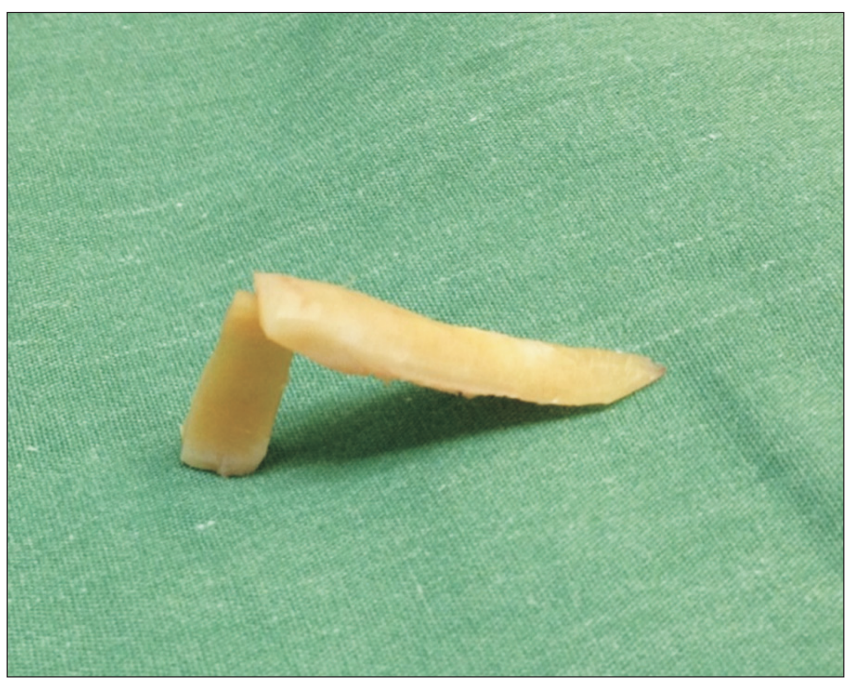

Fig. 18. L-shape reconstruction.

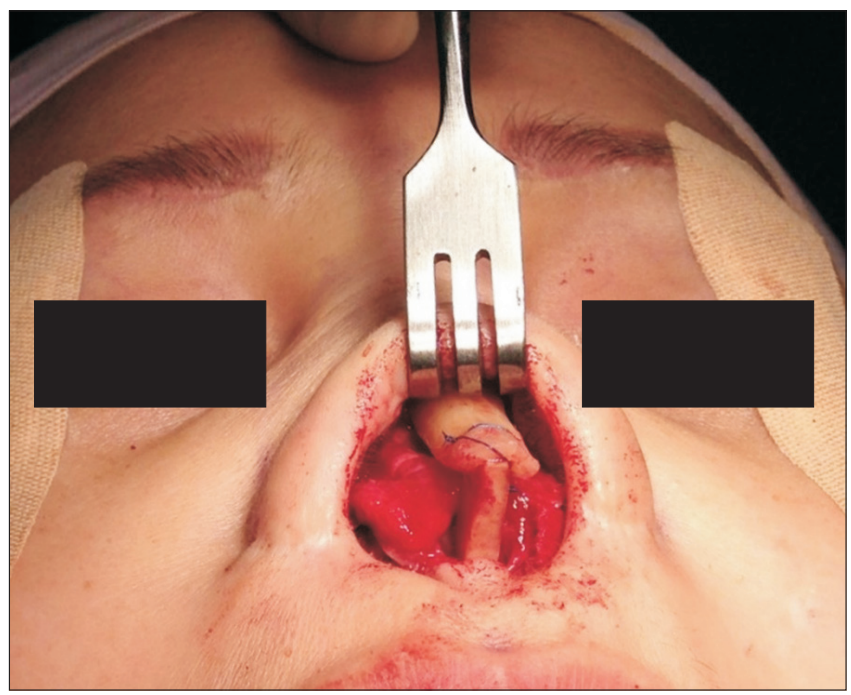

Fig. 19. L-shape reconstruction. of the alar cartilages to the reconstructed septal graft. Lengthening of the nose is part of the augmentation procedure with an L-shaped rib-graft (Fig. 18). The alar domes can be achieved by placing a transplant caudal to the medial crura (Fig. 19). Lengthening may also be achieved by resection of the caudal edge of the cartilaginous septum close to the nasal spine by reducing the nasal labial angle.

\section{Tip definition point and alar contour}

Lengthening and projection the tip are done by shield graft or tip onlay graft (Fig. 20). Combining lower lateral cartilage onlay graft or alar contour graft helps to lower the support of the alar $\operatorname{rim}$ (Fig. 21).

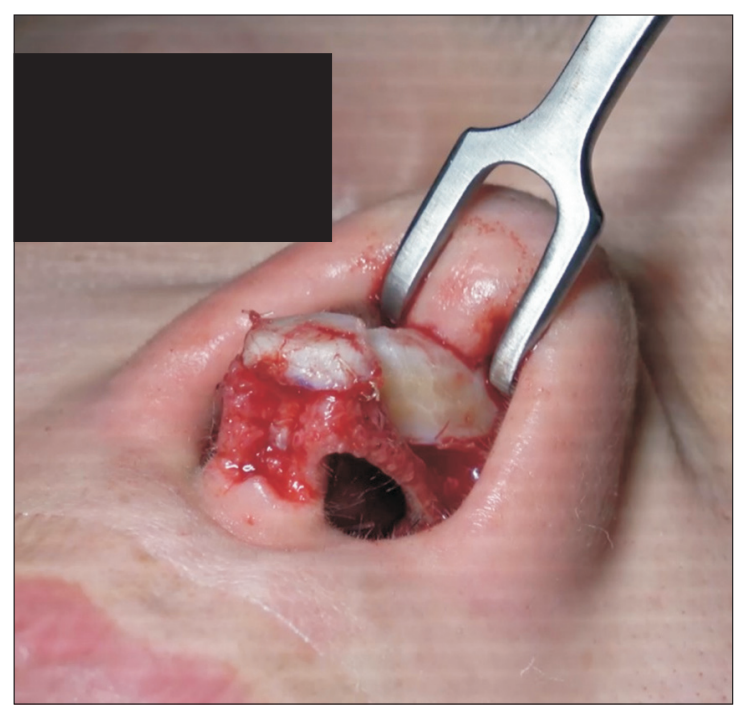

Fig. 20. Tip definition point and alar contour.

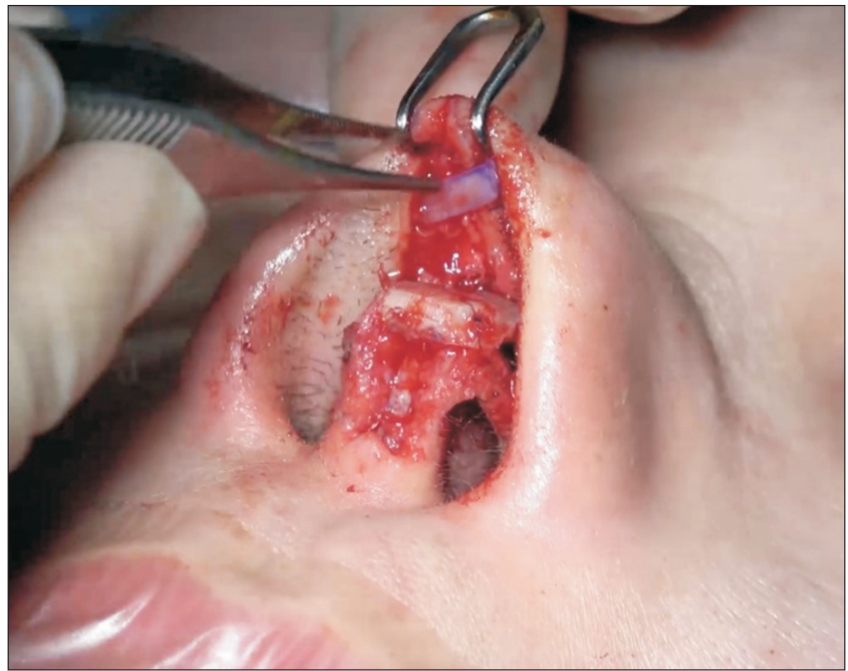

Fig. 21. Tip definition point and alar contour. 


\section{Dorsal Augmentation}

The material used for dorsal augmentation is alloplastic material such as expanded Polytetrafluoroethylene (e-PTFE; Gore-Tex). For dorsal onlay graft, the material is boat-shaped rib cartilage (Fig. 22, 23). For thin skin patients, the cartilage is wrapped with perichondrium or capsular tissue on tip \& dorsal onlay graft.

\section{Results}

From 2013 to 2017, 253 contracted nose correction rhinoplasty procedures were conducted on Asian patients. A satisfactory rate of $95 \%$ (240 cases) was obtained. Thirteen cases (5\%) of

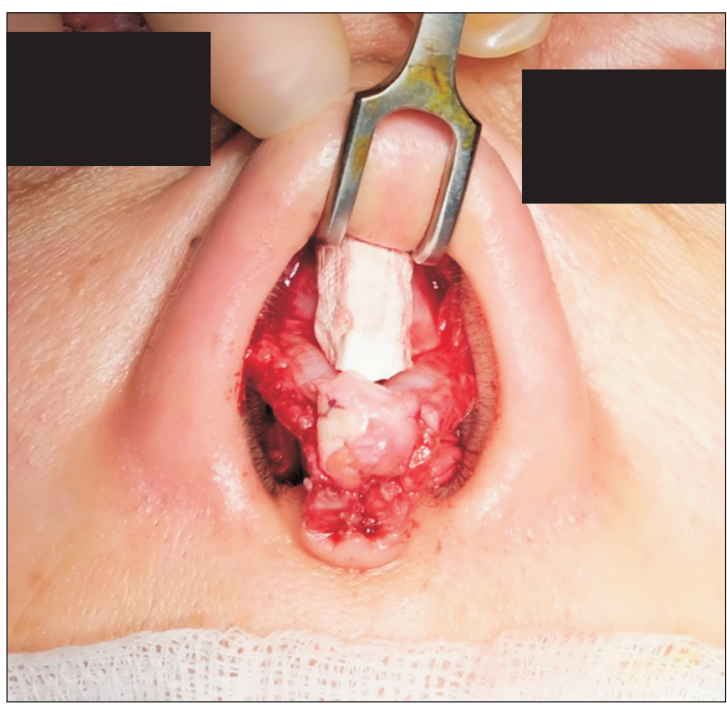

Fig. 22. Dorsal augmentation.

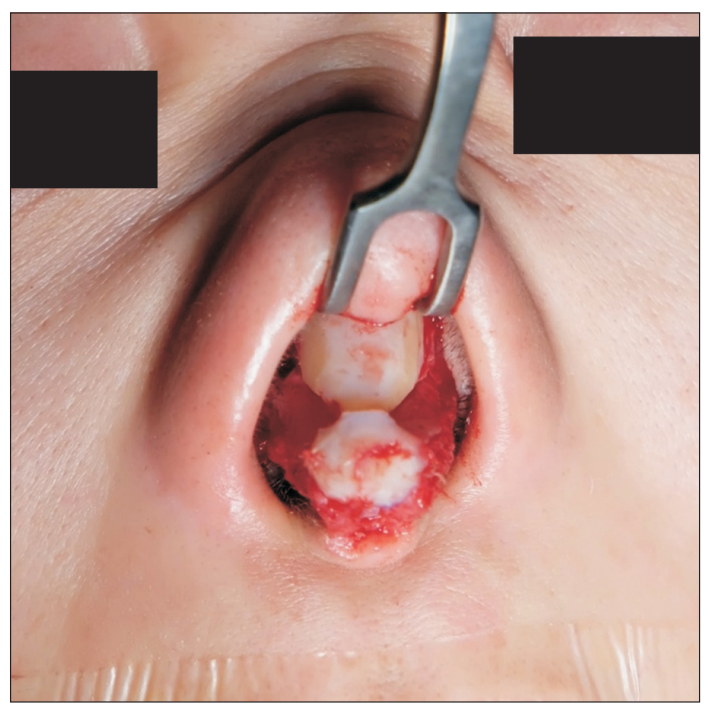

Fig. 23. Dorsal augmentation.
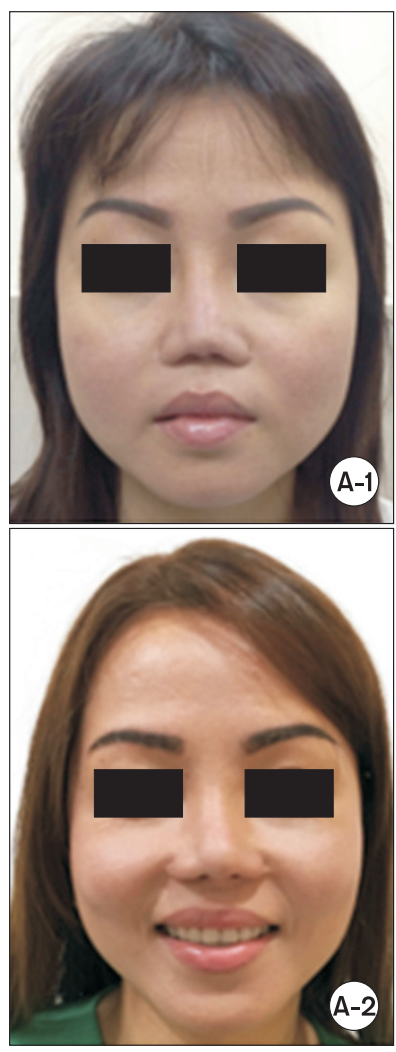
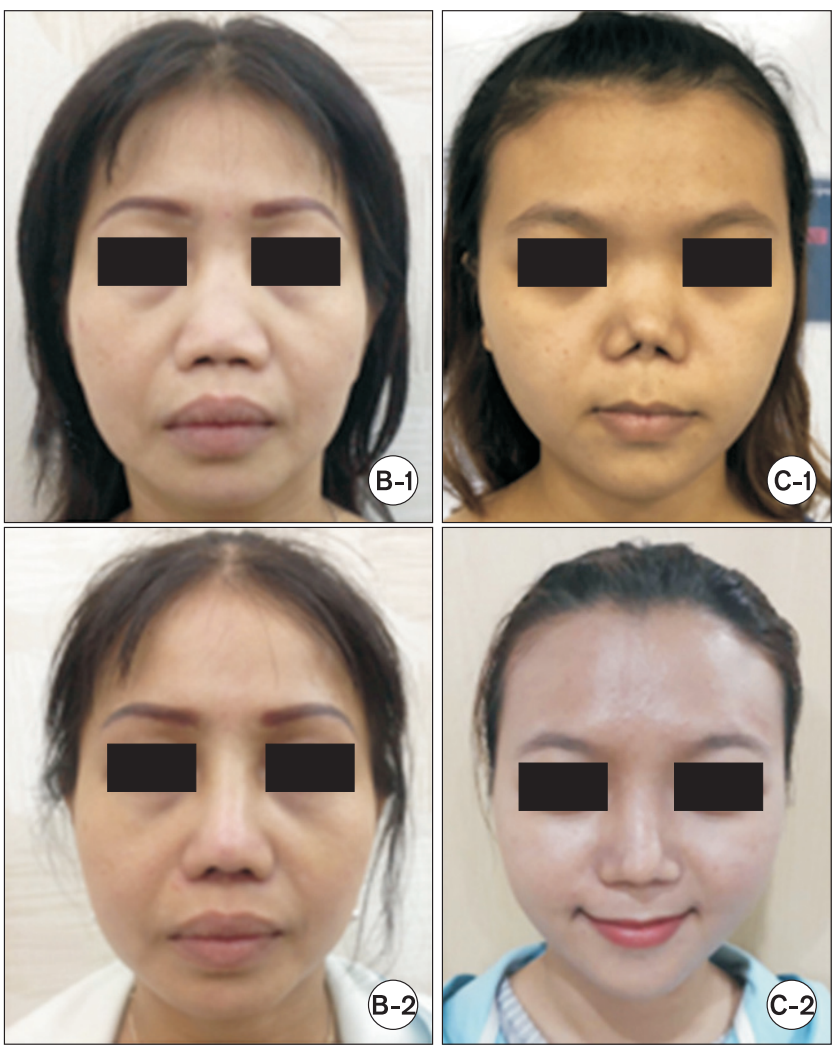

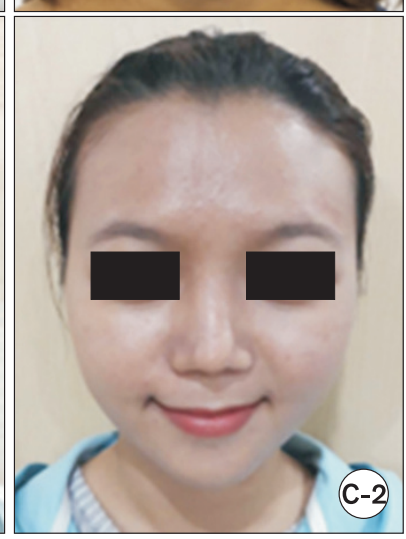

Fig. 24. Frontal view of patients before (A-1, B-1, and C-1) and after (A-2, B-2, and C-2) correction of severely contracted nose, pinched tip, retracted alar, asymmetrical nostril, lost columella or septal cartilage by necrosis due to previous rhinoplasty, excessive alar excision, collapsed external valve, nasal obstruction, asymmetrical silicone implant. 
these required revision surgeries because of the overcorrection or undercorrection of nasal length, poor tip projection, or inadequate correction of columellar retraction.

The first patient (Fig. 24, 25; patient A) was a 31-year-old woman who had already had 7 rhinoplasties with the use of silicone implants. The patient's conditions were as follows: pinched tip, retracted ala, asymmetrical nostril, lost columella by necrosis resulting from previous rhinoplasty, excessive alar excision, collapsed external valve, nasal obstruction, and asymmetrical silicone implant.

The performed procedures were as follows: open rhinoplasty, dual plane dissection, silicone implant removal, rhinoplasty with costal cartilage, lengthening of the nose with L-shaped reconstruction of the dorsal onlay graft and columella strut graft, soft tissue onlay graft of the tip, and alar contour graft. The columella was reconstructed by bilateral alar base flaps, reposition and reconstruction of the ala by V-Y flap, and alar rim excision.

The second patient was a 41-year-old woman (Fig. 24, 25; patient B) and had undergone 8 rhinoplasty operations with silicone implants. The patient's condition was as follows: severely contracted nose, retracted alar, asymmetrical nostril, lost columella and septal cartilage from previous rhinoplasty, excessive alar excision, and collapsed external valve. The performed procedures were as follows: open rhinoplasty, dual plane dissection, rhinoplasty with costal cartilage, lengthening of the nose with L-shaped reconstruction of the dorsal onlay graft and columella strut graft, soft tissue onlay graft of the tip, and alar contour graft. The columella was reconstructed by bilateral alar base flaps; and the alar was repositioned by V-Y flaps and alar rim excision.

The third patient was a 23-year-old woman (Fig. 24, 25; patient C) who had been through 5 rhinoplasty operations and had infection from a silicone implant. The patient's condition was as follows: severely contracted nose, pinched tip, retracted alar, asymmetrical nostrils, lost columella by necrosis from previous rhinoplasty operations, collapsed external valve, and nasal obstruction. The performing procedures were: open rhinoplasty, dual plane dissection, rhinoplasty with costal cartilage, lengthening of the nose, bilateral extended spreader graft and caudal septal extension graft, lateral crural onlay graft combined with alar contour graft, and soft tissue onlay graft of the tip.
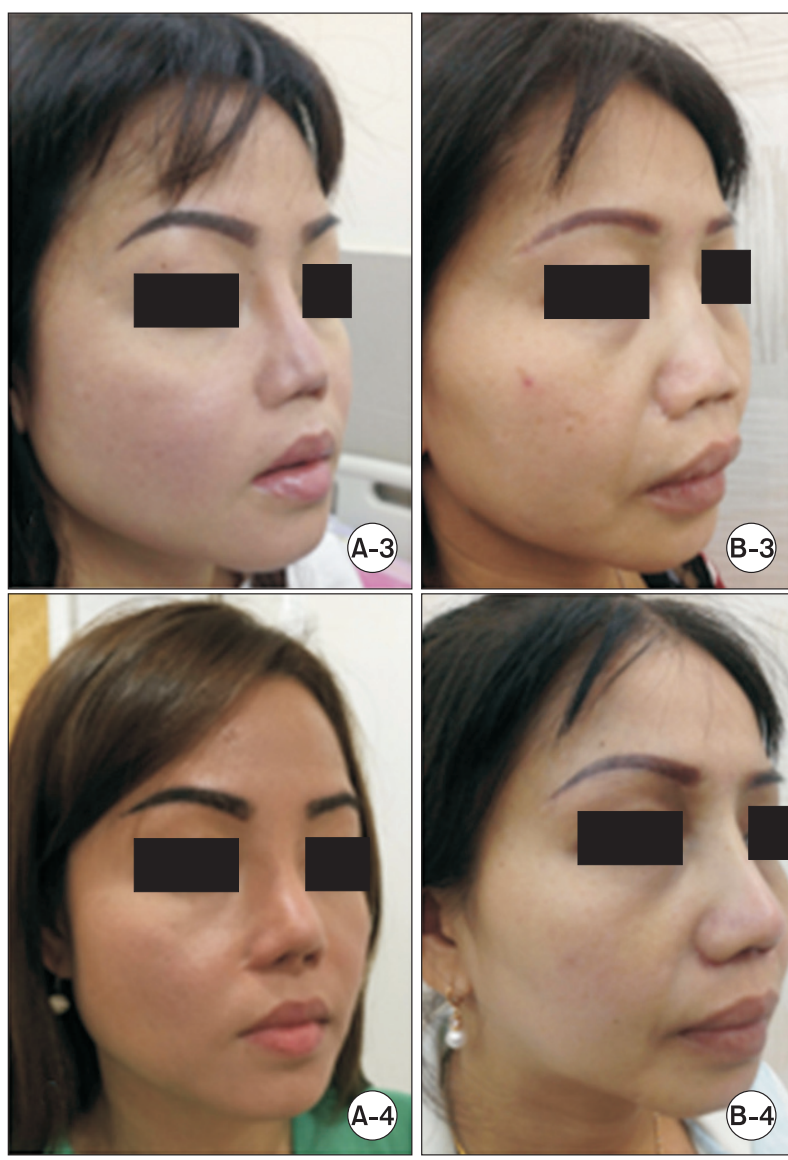

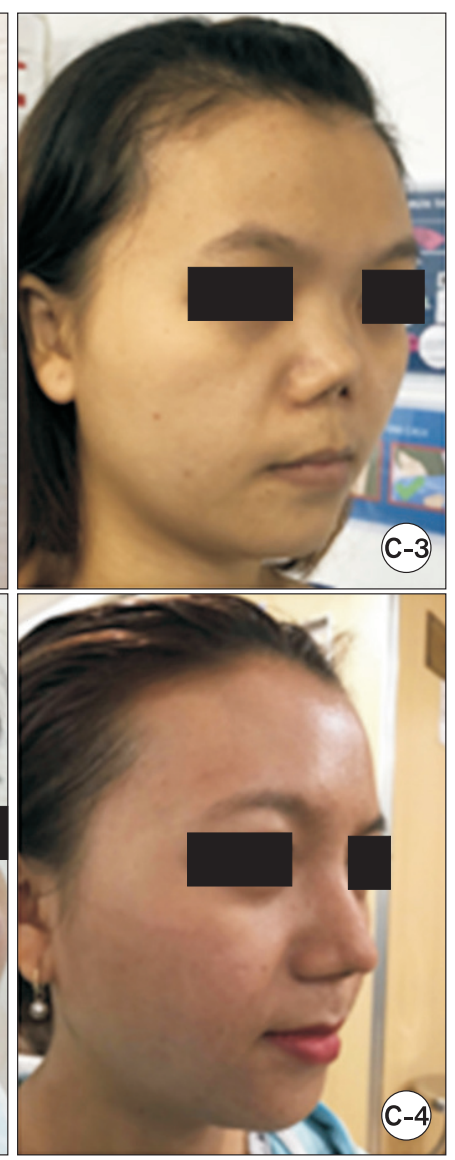

Fig. 25. Oblique view of patients before (A-3, B-3, and C-3) and after (A-4, B-4, and C-4) correction of severely contracted nose, pinched tip, retracted alar, asymmetrical nostril, lost columella, or septal cartilage by necrosis due to previous rhinoplasty, excessive alar excision, collapsed external valve, nasal obstruction, asymmetrical silicone implant. 
All the three mentioned cases were highly successful with very little to no complications postoperatively.

\section{Discussion}

Dual plane dissection technique was used in all 3 typical cases that were presented in this paper and all other cases. The technique allows skin flap advancement, which is essential for skin lengthening. This dissection also fully releases the adherence between skin-scar/capsule flap-deep planes (periosteum). Although this technique is highly helpful, its main potential complications include surface irregularity, necrosis, and dimpling. In the case of revision rhinoplasty, contraction of scar tissues deforms the shape of the nasal tip, especially if the patient's nose is too short, which increases the risk of getting related complications. My preventive procedure was to ensure only dissecting 1 skin layer on 1 surface. If the dissected layer was too thin, insufficient blood supply would occur, which could potentially lead to necrosis. Moreover, if the extracted layer was uneven, surface irregularity would appear. However, these complications rarely occur in my experience, only $3 \%$ to $5 \%$ of cases requiring this procedure had one of these complications.

In the contracted nose correction procedure, insufficient blood supply or necrosis of the skin flap occasionally happens when elongation is performed due to high tension. In order to prevent this from happening, I released the adherence on a larger area to reduce the tension. The soft tissue layers at the nasal tip and columella were preserved to prevent direct contact between the dermis and the grafting material. Only necrosis at the inframarginal rim was observed, but it healed itself.

Although contraction is often caused by alloplastic implants, I hardly observed it when performing over 250 contracted nose correction cases. In my experience, Gore-tex and autogenous costal cartilage materials actually reduce the risk of contraction and infection, and have better biocompatibility. Gore-tex has its own history in its application in many surgical specialties, such as vascular surgery, and has shown outstanding biocompatibility [4]. Maas et al. [5] also reported that in an animal model, e-PTFE had minimal inflammatory response combined with an increase in stability over time in the body, which is an exceptional implant characteristic.

To conclude, contracted nose commonly occurs as a result of a long-term rhinoplasty using silicone implant with multiple previous surgeries or infection that damages the soft tissue structure.

The length of the nose can be extended by dual plane dissection to release adhesion of skin and scar tissue, extended spreader grafting, dorsal augmentation, and tip and alar grafting.

The goal of the surgery is to perform proper surgical techniques and a combination of procedures to bring out the best outcomes for both patients and surgeons.

\section{Conflicts of interest}

The author has nothing to disclose.

\section{References}

1. Wu WT. The Oriental nose: an anatomical basis for surgery. Ann Acad Med Singapore 1992;21:176-89.

2. Jung DH, Kim BR, Choi JY, Rho YS, Park HJ, Han WW. Gross and pathologic analysis of long-term silicone implants inserted into the human body for augmentation rhinoplasty: 221 revision cases. Plast Reconstr Surg 2007;120:1997-2003.

3. Matsunaga RS. Augmentation rhinoplasty of Asian noses. Facial Plast Surg Clin North Am 1996;4:75-85.

4. McAuley CE, Steed DL, Webster MW. Seven-year follow-up of expanded polytetrafluoroethylene (PTFE) femoropopliteal bypass grafts. Ann Surg 1984;199:57-60.

5. Maas CS, Gnepp DR, Bumpous J. Expanded polytetrafluoroethylene (Gore-Tex soft-tissue patch) in facial augmentation. Arch Otolaryngol Head Neck Surg 1993;119:1008-14. 